\title{
RESUMEN
}

La instrucción pastoral "Communio et Progressio“ da decisivos principios según la opinión del autor, para arreglar las relaciones entre las organizaciones profesionales católicas del periodismo y los centros eclesiásticos para los medios de la comunicación social. Trata de mostrar a base de la Unión Católica Internacional de la Prensa (UCIP) como la libertad de las reuniones libres es respetada, pero como la Iglesia por otra parte no puede estar desinteresada de influir sobre la "actividad periodística de los católicos". Sin embargo las organizaciones „de la“ Iglesia dependiente de la jerarquía y las asociaciones de profesionales católicos „en“ la Iglesia son diferenciadas. El autor saca una multitud de conclusiones importantes.

\section{Forderungen der Pastoralinstruktion an Hierarchie und Klerus}

\author{
von Otto Kaspar
}

Wer behauptet, die Kirche habe ein gestörtes Verhältnis zu den Massenmedien, spricht eine Platituide aus. Manche behaupten sogar, sie habe gar keines. Das ist gewiß übertrieben. Vorbild und Pionier, wie auf anderen Sektoren - des Gesundheitsund Bildungswesens etwa - war aber die Kirche bei den "Instrumenten der sozialen Kommunikation" gewiß nicht.1 Das zeigt sich nicht zuletzt am Interesse bzw. Desinteresse, das die Pastoralinstruktion "Communio et Progressio"2 erfahren hat.

Nachdem laut geklagt worden war, wie schwach und unzureichend das Konzilsdekret über die Medien ${ }^{3}$ ausgefallen sei, hatten die an den Medien interessierten Katholiken angenommen, daß dieses neue Ausführungsdokument offene Ohren und Herzen finden werde (von wegen des "Beherzigens"). Nun ist aber "Communio et Progressio" bereits über ein halbes Jahr veröffentlicht. Wie ist das Echo? Gleichsam Null.

Kirchliche Behörden, kirchliche Organisationen, kirchliche Orden und Gesellschaften scheinen oft noch gar nicht zu wissen, daß es ein solches Dokument gibt, das zum Teil "unerhört" ist, einfach weil man ein so offenes Verhältnis der Kirche zu den

Dr. Otto Kaspar ist Chefredakteur des „Ruhr-Wort“, Essen, Begründer der Entwicklungskommission der Katholischen Weltunion der Presse (UCIP) sowie "geistiger Vater" und Initiator des "Catholic Media Council“ (siehe S. 57-63 dieser Ausgabe). 
Instrumenten der sozialen Kommunikation bisher weder zu Gesicht bekommen noch überhaupt von einer solchen Haltung in der Kirche gehört hat. Man kann sich vielleicht damit trösten, daß es erfahrungsgemäß in der Kirche sehr lange dauert, bis sich etwas in ihr entwickelt, entfaltet und angenommen wird. Dem entspricht, daß einmal akzeptierte Einrichtungen und Praktiken sich selbst dann noch beharrlich am Leben halten, wenn sie für das Leben der Kirche längst zum Ballast geworden sind.

Also soll man geduldig versuchen, dieses wichtige Nachfolgedokument des Konzils zu propagieren. Im folgenden wird nur versucht, jene Stellen von "Communio et Progressio " zu beleuchten, die für die geforderte neue Haltung der Kirche gegenüber den Massenmedien - der Gläubigen insgesamt und der geistlichen Berufe im besonderen - wichtig sind.

Die Pastoralinstruktion bemüht sich erst gar nicht, um Verständnis zu werben, daß die Massenmedien einen Wert haben - sie geht davon aus: „Was in dieser Instruktion an Grundsätzlichem angesprochen wird, sollte heute Gemeingut aller Menschen sein"4, heißt es. „Da die Kommunikationsmittel oft die einzigen Nachrichtenverbindungen $z$ wischen Kirche und Welt sind, würde jemand, der sie ungenützt läßt, von Gott geliehene Talente in die Erde vergraben. " 5 Diese Feststellung ist ein lapidarer Folgesatz aus der allgemeinen Zustimmung zu den Kommunikationsmitteln.

Es versteht sich, daß nicht nur eine eigene „Medienerziehung “ in der Erwachsenenbildung und eine "Medienpädagogik" empfohlen werden, weil „Vertrautheit" und "direkter Umgang mit den Kommunikationsmitteln " vorausgesetzt sind. ${ }^{6}$ Es werden auch „Eltern, Erzieher, Priester und katholische Organisationen“ zur „Förderung begabter Kandidaten" aufgefordert; dafür sei ein „entsprechender Fonds einzurichten ". ${ }^{7}$

Die Frage "Wer soll das bezahlen?" wird beantwortet: "Angesichts der wachsenden Bedeutung der Instrumente der sozialen Kommunikation für die Menschheit überhaupt und für das Leben der Kirche im besonderen werden es die Bischofskonferenzen als ihre vordringliche Pflicht ansehen, in ihrer pastoralen Gesamtplanung dem Einsatz im Bereich der Kommunikation anders als früher nun einen zentralen Platz einzuräumen. Sie werden für ihren eigenen Verantwortungsbereich sowie für den Bedarf auf internationaler Ebene ausreichende Geldmittel bereitstellen." 8

Besonderes Gewicht für „künftige Priester und Ordensleute, auch Ordensschwestern“ hat ein eigener Abschnitt. Sie müssen, heißt es, „während ihrer Ausbildung mit der Rolle und Auswirkung dieser Medien in der menschlichen Gesellschaft vertraut gemacht und in den Gebrauch dieser Technik eingewiesen werden; sonst blieben sie völlig lebensfern und für ihr späteres Apostolat ungeeignet. Die Vermittlung solcher Kenntnisse muß integraler Bestandteil ihrer Ausbildung sein. Ohne diese Voraussetzung kann überhaupt niemand mehr wirksam apostolisch tätig werden in einer Welt, die täglich stärker von diesen Medien geprägt wird. Weiterhin sollen Priester und Ordensleute die Prozesse der Meinungsbildung genau kennen und sich den Gegebenheiten unserer Zeit anpassen. Denn das Wort Gottes ist den Menschen von heute zu verkünden, und schließlich leisten die Medien selbst einen wirksamen Dienst für die Verkündigung. Wer aus dem Nachwuchs für Priester- und Ordensberufe besondere Neigung und Befähigung zur Arbeit in den Kommunikationsmitteln zeigt, soll dazu weiter ausgebildet werden." $\theta$

Diese harte Erklärung, Priester und Ordensleute blieben nicht nur "völlig lebensfern", und auch "für ihr späteres Apostolat ungeeignet", wenn sie nicht mit den 
Medien vertraut gemacht werden, ergänzt die Formulierung: "Man kann sich nur schwer vorstellen, wie jemand das Gebot Christi treu erfüllen wird, wenn er die Vorteile und Chancen dieser Medien, einer außerordentlich großen Zahl von Menschen die Lehren und Gebote des Evangeliums zu bringen, ungenützt läßt."

Das gilt nicht nur für die sogenannte entwickelte Welt, sondern besonders auch für die Kirche in der Dritten Welt, denn "unter den zahlreichen Formen internationaler Zusammenarbeit, die durch das Wesen der Kommunikationsmittel geradezu aufgedrängt wird, ist die Hilfe für Entwicklungsländer von besonderer Bedeutung. Es ist zugleich Wirkung und Ursache der Unterentwicklung, wenn moderne Kommunikationsmittel nur in unzureichendem Maße vorhanden sind oder ganz fehlen. Ohne diese Techniken kann heute kein Volk mehr die notwendige Information und Bildung erreichen. Von daher wäre der wirtschaftliche, soziale und politische Fortschritt der Entwicklungsländer in Frage gestellt. "11 Angesichts der Zielstrebigkeit, die die Pastoralinstruktionen kennzeichnet, erwächst die Forderung - an die Adresse der Weltkirche gerichtet - wie von selbst: „Den Bischöfen in den Entwicklungsländern müßten unbedingt hinreichend Hilfsmittel zur Verfügung gestellt werden, damit sie den publizistischen Nachwuchs im eigenen Land sachgerecht ausbilden und in die praktische Arbeit einführen lassen können." 12

Diese wenigen Stellen der Pastoralinstruktion sollten jene, die in der Kirche Verantwortung tragen, aufhorchen lassen. Denn was hier über das Verhältnis der Christen zu den Massenmedien gesagt wird, trifft gerade sie gemäß dem Wort: „Wer Ohren hat zu hören, der höre." ${ }^{13}$ Dieses Wort war niemals als „freies Angebot", als luftige Eventualität $z$ wischen einem Ja und Nein nach Lust oder Laune gemeint, sondern als Appell, dessen Beantwortung über Leben und Tod entscheiden werde.

Wird dieser Appell zum Umdenken - das ist nämlich der Grundzug der Pastoralinstruktion „Communio et Progressio" - befolgt werden?

Anmerkungen:

1. Der Verfasser hat bereits vor Erscheinen der Pastoralinstruktion „Communio et Progressio" auf Wunsch von Ordensgeneralaten ein Exposé zum Thema "Der ,Medienmissionar" als neuer Berufstyp" gemacht, das in der nächsten Ausgabe dieser Zeitschrift veröffentlicht wird.

2. Päpstliche Kommission für die Instrumente der Sozialen Kommunikation: Pastoralinstruktion "Communio et Progressio" über die Instrumente der Sozialen Kommunikation (lateinisch-deutsch), veröffentlicht im Auftrag des II. Vatikanischen Okumenischen Konzils. Von den deutschen Bischöfen approbierte Ubersetzung, kommentiert von Hans Wagner in: „Nachkonziliare Dokumentation“, Bd. 11, 1971 (Paulinus-Verlag Trier). Zitiert: Pastoralinstruktion.

3. Dekret über die sozialen Kommunikationsmittel, in: „Lexikon für Theologie und Kirche“: Das Zweite Vatikanische Konzil I, 1966 (Herder, Freiburg, Basel, Wien). 391 Seiten, hier: S. 112-135.

4. Pastoralinstruktion, Abschnitt 64.

5. Pastoralinstruktion, Abschnitt 123.

6. Pastoralinstruktion, Abschnitt 110.

7. Pastoralinstruktion, Abschnitt 109.

8. Pastoralinstruktion, Abschnitt 134.

9. Pastoralinstruktion, Abschnitt 111.

10. Pastoralinstruktion, Abschnitt 126.

11. Pastoralinstruktion, Abschnitt 92; über die Pastoralinstruktion im Verhältnis zur Dritten Welt erscheint in dieser Zeitschrift ein eigener Beitrag.

12. Pastoralinstruktion, Abschnitt 109.

13. Mt 11,15 . 


\section{S U M M A R Y}

The reaction of the public to the pastoral instruction "Communio et Progressio" has, it seems, been limited even in church circles. Consequently this important follow-up document of the Council will have to be patiently propagated. Only those parts of the document will be spotlighted here which are important for the attitude of the church towards the mass media. This means among other things that such harsh statements have to be made as: priests and clerics remain completely shut off from reality and unsuited for their later mission if they are not familiarised with the media. The harsh demands which the pastoral instruction makes on the church hierarchy and clergy ought to become a matter of concern for all those with positions of responsibility in the church. For the pastoral instruction is essentially, no more or less than a call to rethink.

\section{R E S U M E N}

El eco del público a la instrucción pastoral "Communio et Progressio“ es aparentemente pequeño en círculos eclesiásticos mismos. Por eso este documento importante siguiendo al Concilio podrá estar propagado pacientemente. Aquí solamente están examinados esos pasajes del documento, que son importantes para la nueva actitud de la Iglesia con los medios de masas. De eso resultan entre otras cosas declaraciones tan rigurosas como éstas: que sacerdotes y religiosos quedan „enteramente lejos de la vida“ y también „impropios para su apostolado posterior", si no son hecho versados en los medios. Todos los responsables en la Iglesia deberían escuchar con atención a las rigurosas demandas que la instrucción pastoral hace a la jerarquía y al clero. Porque la instrucción pastoral en su rasgo esencial no es ni más ni menos que una llamada para repensarse. 